EPJ Web of Conferences 70, 00056 (2014)

DOI: 10.1051/epjconf/ 20147000056

(C) Owned by the authors, published by EDP Sciences, 2014

\title{
Generation of high-energy particles, neutrino and fotons in magnetosphere of collapsing star
}

\author{
Volodymyr Kryvdyk \\ Faculty of Physics, Taras Shevchenko national university of Kyiv \\ 64/13, Volodymyrska Street, City of Kyiv, Ukraine, 01601 \\ Corresponding author: kryvdyk@gmail.com
}

\begin{abstract}
The generation of particles, photons and neutrinos in magnetosphere of collapsing star are considered. These processes are caused the self-interaction initial accelerating in magnetosphere protons and electrons. The second particles and photons will arise as a result of this self-interaction, which in turn will generate charged particles, photons and neutrino (cascade process). These processes are especially effective for the formation collapsing star magnetosphere from the secondary charged particles. In addition, the particles, photons and neutrinos will be generated in magnetosphere of collapsing star as result of these processes.
\end{abstract}

\section{Introduction}

The initial magnetospheres of collapsing star consist with protons and electrons. These particles accelerate during collapse to relativistic energy $[1,2,3,4,5]$. Interacting among themselves and the magnetic fields in magnetosphere, these particles will lose their energy on the ionization and radiation. Electrons will be loss the energy with the most speed, and therefore the electrons lifetime is substantially smaller from the protons lifetime. As result the initial electrons will be loss their energy very fast. At the same time in the magnetosphere of collapsing stars will generate the secondary particles namely electrons, protons, neutrons, mesons, neutrino and photons. During the cascade interaction the secondary particles will generate the other particles and photons. Thus on the later stage of collapse the magnetosphere of collapsing stars consist with the particles and photons generating by multiple interaction of particles.

In this paper we will consider in detail the mechanisms of the generation of the particles, photons and neutrino in the magnetosphere of collapsing stars.

\section{Generation of the high energy neutrino, particles and photons in magnetosphere of collapsing star.}

In this subsection we consider the mechanisms of the generation the secondary particles and photons by nuclear interaction in magnetosphere. Especially important are the cascade showers of the charged particles, neutrino and photons, generating in magnetosphere. These processes are especially effective for the formation of magnetosphere of collapsing star.

In the magnetosphere of collapsing star can be generated the next secondary charged particles, neutrino and photons:

1. $\pi$ - mesons, $\mu$ - mesons, electrons, positrons and neutrino by nuclear interaction.e.

2. Electrons-positron pairs in nuclear field by means of gamma-rays.

3. Electrons-positron pairs as result of the collision of the charged particles.

4. Electrons-positron pairs as result of the collision of two photons.

5. $\mu$ - mesons pairs by passing of gamma -rays in the nuclear field.

This is an Open Access article distributed under the terms of the Creative Commons Attribution License 2.0, which permits unrestricted use, distribution, and reproduction in any medium, provided the original work is properly cited. 
6. The recoil electrons by collision of the charged particles.

7. Neutrino by the collision of the charged particles and nuclear interaction in magnetosphere.

In magnetosphere of collapsing star can be generated of the charged particles, neutrons, neutrino and photons as result these processes. The scheme of generation these particles are given in Table 1.

Table 1. Particles generation in the magnetosphere collapsing star

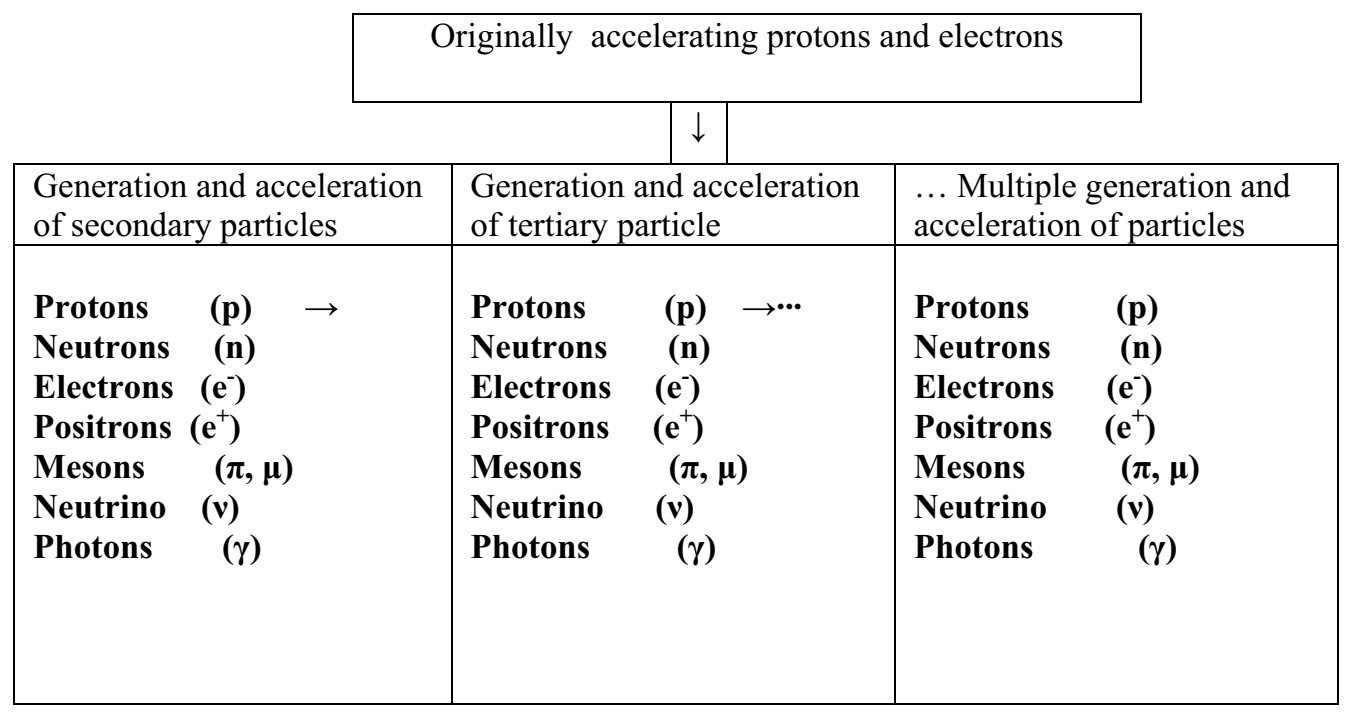

Thus the charged particles, neutrons, neutrino and photons will generate uninterruptedly in the magnetosphere of collapsing star. We will consider these processes in next subsection.

\section{Generation of $\pi$-mesons, $\mu$-mesons, electrons, positrons and neutrino by nuclear interaction in magnetosphere of collapsing star.}

In previously author papers $[1,2,3,4,5]$ have been should that protons and electrons will accelerate in magnetosphere of collapsing star to relativistic energy. The accelerating protons will interact with other protons in magnetosphere and will generate mesons by next nuclear interactions [6, $7,8]$

$$
\begin{gathered}
p+p \rightarrow p+p+n_{1}\left(\pi^{+}+\pi^{-}\right)+n_{2} \pi^{0} \\
p+p \rightarrow p+n+\pi^{+}+n_{3}\left(\pi^{+}+\pi^{-}\right)+n_{4} \pi^{0} \\
p+p \rightarrow n+n+2 \pi^{+}+n_{5}\left(\pi^{+}+\pi^{-}\right)+n_{6} \pi^{0} \\
p+p \rightarrow D+\pi^{+}+n_{7}\left(\pi^{+}+\pi^{-}\right)+n_{8} \pi^{0}
\end{gathered}
$$

Here $p$ - proton, $n$ - neutron, $D$ - deuteron, $n_{1^{-}} n_{2^{-}}$positive integers.

In the later the second electrons, positrons, photons and neutrino will be generated by decay of mesons

$$
\begin{gathered}
\pi^{ \pm} \rightarrow \mu^{ \pm}+v_{\mu} / \bar{v}_{\mu} \\
\pi^{0} \rightarrow \gamma+\gamma \\
\mu^{ \pm} \rightarrow e^{ \pm}+v_{e} / \bar{v}_{e}+v_{\mu} / \bar{v}_{\mu}
\end{gathered}
$$


For relativistic protons with energy from $290 \mathrm{MeV}$ to $1 \mathrm{\Gamma eV}$ only one with reaction can be possible [6]

$$
p+p \rightarrow p+n+\pi^{+} \text {, or } p+p+\pi^{0}, \text { or } D+\pi^{+}
$$

The minimum energy $E_{\min }$ for the generation $\pi$-mesons by nuclear proton is

$$
E_{\text {min }}=\frac{Y^{2} m_{\pi}^{2} c^{4}}{2 m_{p} c^{2}}+2 Y m_{\pi} c^{2} \approx Y(280+10 Y)[\mathrm{MeB}] .
$$

The generation $\pi$-mesons is possible only for protons with high energy more $290 \mathrm{MeV}$. These protons accelerate in the variable magnetic fields in the magnetosphere of collapsing star [1-5]. Therefore we can assert that the reactions (1) and (2) can generated the powerful flux secondary electrons, positrons, gamma-rays and neutrino in magnetospheres by gravitational collapse. Thus the collapsing stars will the powerful source of neutrino, cosmic rays and the nonthermal electromagnetic radiation.

In addition the charged particles, the neutral neutrons will be generated by reactions (1) and (2). These particles not interact with the magnetic fields and therefore their will pass free in magnetosphere forming the neutron wind around collapsing star. When in magnetosphere are the sufficiently value of free neutrons and protons, neutrino can generated by urca-processes when neutrons and protons capture of electron or positron and neutrino radiate by reactions [9]

$$
\begin{aligned}
& p+e^{-} \rightarrow n+v_{e} \\
& n+e^{+} \rightarrow p+\bar{v}_{e}, \\
& n \rightarrow p+e^{-}+\bar{v}_{e}
\end{aligned}
$$

Presence of the strong magnetic fields can influence strong on the velocity urca-reactions. In papers $[10,11]$ was should that in the very strong magnetic fields $\left(H \gg 4 \cdot 10^{13} \mathrm{Gs}\right)$ the velocity betadecay and urca- processes can arise in hundred time.

If the big value free neutrons are in the stable equilibrium with the small value protons and electrons, then the neutrons scattering result to modify urca-reactions $[12,13]$

$$
\begin{gathered}
n+n \rightarrow n+p+e^{-}+\bar{v}_{e}, \\
n+p+e^{-} \rightarrow n+n+v_{e},
\end{gathered}
$$

If Fermi energy for neutrons exceed the rest energy of muon $m_{\mu} c^{2}$, then in result modify urcareactions the muon neutrino will be generated

$$
\begin{gathered}
n+n \rightarrow n+p+\mu^{-}+\bar{v}_{\mu} \\
n+p+\mu^{-} \rightarrow n+n+v_{\mu} .
\end{gathered}
$$

The reaction with muon neutrino can occur only in the very density medium in density $\rho \geq 8 \cdot 10^{14} \mathrm{~g} / \mathrm{sm}^{3}$ , that exceed the nuclear density $\rho_{\text {nuc }} \approx 3.7 \cdot 10^{14} \mathrm{~g} / \mathrm{sm}^{3}$. In addition to muon neutrino, in magnetosphere are also quasi- free pions (see reactions (1) and (2)), therefore in magnetosphere is possible also one class urca-reactions as a result of which of neutrinos, electrons and pions can be generated:

$$
\begin{aligned}
& \pi^{-}+n \rightarrow n+e^{-}+\bar{v}_{e} \\
& n+e^{-} \rightarrow n+\pi^{-}+v_{e} \\
& \pi^{-}+n \rightarrow n+\mu^{-}+\bar{v}_{\mu} \\
& n+\mu^{-} \rightarrow n+\pi^{-}+v_{\mu} .
\end{aligned}
$$




\section{Generation of electron-positron pairs in magnetosphere of collapsing star.}

The accelerating in magnetosphere of collapsing star initial electrons will lose their energy during gravitational collapse in consequence of the bremsstrahlung losses. Therefore for the existence of magnetosphere is necessary the continuous generation of the new secondary electrons by the interactions photons and particles in magnetosphere. These questions will are considered in the next subsections.

\subsection{Generation of electron-positron pairs by interaction the gamma- photons with nuclear.}

The electron-positron pairs will generate during passing of gamma - photons with energy $h v>$ $2 m_{\mathrm{e}} \mathrm{c}^{2}=1.022 \mathrm{MeB}$ in Coulomb field of nuclear with charge $e Z$. Here $m_{e} c^{2} \approx 0.511 \mathrm{MeB}$ is electron rest energy. For photons with energy $h v \gg m_{\mathrm{e}} \mathrm{c}^{2}$ the nuclear process cross-section for generation of electron-positron pairs is [6]

$$
\begin{array}{lll}
\sigma(h v)=4 \alpha Z^{2} r_{0}^{2}\left[\frac{7}{9} \ln \left(\frac{2 h v}{m_{\mathrm{e}} c^{2}}\right)-\frac{109}{54}\right] & \text { for } & h v \ll \frac{m_{\mathrm{e}} c^{2}}{\alpha Z^{1 / 3}} \\
\sigma(h v)=4 \alpha Z^{2} r_{0}^{2}\left[\frac{7}{9} \ln \left(\frac{191}{Z^{1 / 3}}\right)-\frac{1}{54}\right] & \text { for } & h v \gg \frac{m_{\mathrm{e}} c^{2}}{\alpha Z^{1 / 3}} .
\end{array}
$$

Here $r_{0}=e^{2} / m_{\mathrm{e}} \mathrm{c}^{2} \approx 2.8 \cdot 10^{-13} \mathrm{sm}$ is classical electron radius; $\alpha \approx 1 / 137$ is fine structure constant. The value $4 \alpha r_{0}^{2} \approx 2.318 \cdot 10^{-27} \mathrm{sm}^{2}$.

Since the main nuclear in magnetosphere are protons $(Z=1)$, the interaction cross-section for the generation of electron-positron pairs by the interaction the gamma- photons with protons in magnetosphere of collapsing star are

$$
\begin{array}{lll}
\sigma_{p h p}(h v)=2.318 \cdot 10^{-27}\left[\frac{7}{9} \ln \left(\frac{2 h v}{m_{\mathrm{e}} \mathrm{c}^{2}}\right)-\frac{109}{54}\right] & \text { for } & h v \ll 70 \mathrm{MeV}, \\
\sigma_{p h p}(h v)=2.318 \cdot 10^{-27}\left[\frac{7}{9} \ln (191)-\frac{1}{54}\right] & \text { for } \quad h v \gg 70 \mathrm{MeV} .
\end{array}
$$

The numerical values of nuclear cross-section are

$$
\begin{aligned}
& \sigma_{p h p}(h v) \approx 4.158 \cdot 10^{-28} \mathrm{~cm}^{2} \quad \text { for } \quad h v \ll 70 \mathrm{MeV} \text {, } \\
& \sigma_{p h p}(h v) \approx 9.426 \cdot 10^{-27} \mathrm{~cm}^{2} \quad \text { for } h v \gg 70 \mathrm{MeV} \text {. }
\end{aligned}
$$

How see, the nuclear interaction cross-section weak depend on the photons energy.

\subsection{Generation of electron-positron pairs by the interaction of charged particles}

Magnetosphere of collapsing star consists basically with proton- electron plasma. By the mutual interaction of charged particles will generate the electron-positron pairs. The cross-section for this interaction is [6]

$$
\sigma_{e p}(h v)=\frac{28}{27 \pi} \alpha^{2} r_{0}^{2} \ln (1 / \alpha)\left[3 \ln \left(\frac{\mathrm{E}}{\mathrm{m}_{\mathrm{e}} \mathrm{c}^{2}}\right) \ln \left(\frac{\mathrm{E}}{191 \mathrm{~m}_{\mathrm{e}} \mathrm{c}^{2}}\right)+\ln ^{2}(191)\right] .
$$


Substituting a numerical values $\alpha \approx 1 / 137$ and $r_{0} \approx 2.8 \cdot 10^{-13} \mathrm{sm}, m_{e} c^{2} \approx 0.511 \mathrm{MeV}$ we obtain

$$
\sigma_{e p}(h v) \approx 6.784 \cdot 10^{-30}\left[3 \ln (2 E) \ln \left(10^{-2} E\right)+27.6\right]
$$

Here $E$ is energy in $\mathrm{MeV}$.

This cross-section not depend on the electron energy, and their numerical values is

$$
\sigma_{e p}(h v) \approx 6.3 \cdot 10^{-29} \mathrm{~cm}^{2}
$$

\subsection{Generation of electron-positron pairs by collision two photons.}

Electron-positron pairs in magnetosphere can be generated also by mutual collision two photons. These photons arise when two photons with energy $E_{1}$ and $E_{2}$ (by condition $E_{l} E_{2}>\left(m_{e} c^{2}\right)^{2}$. Here $m_{e}$ $c^{2} \approx 0.511 \mathrm{MeV}$ - the energy rest electron mass. Cross-section for this interaction is $[6,14]$

$$
\sigma_{2 p h}\left(E_{1}, E_{2}\right)=\frac{\pi r_{0}^{2}}{2}\left(1-\beta^{2}\right)\left[2 \beta\left(\beta^{2}-1\right)+\left(3+\beta^{4}\right) \ln \left(\frac{1+\beta}{1-\beta}\right)\right]
$$

Here $\beta=\left[1-\frac{\left(m_{e} c^{2}\right)^{2}}{E_{1} E_{2}}\right]^{1 / 2}, \quad \beta c$ - electron velocity in center-of-mass system. Value $\sigma_{2 p h}\left(E_{1}, E_{2}\right)$ change in range

$$
2.9 \cdot 10^{-25} \leq \sigma_{2 p h}\left(E_{1}, E_{2}\right) \leq 5.2 \cdot 10^{-26} \mathrm{sm}^{2} \quad \text { for } 0.85 \leq \beta \leq 0.999 .
$$

The cross-section for this interaction exceeds the cross-section in sections 3.1 and 3.2 , therefore this process may be the most effective process for the formation of electron-positron pairs in the magnetosphere.

\section{Generation $\mu$-meson pairs in nuclear fields by gamma-rays.}

If the energy of gamma photon exceed a threshold energy $2 m_{\mu} c^{2} \approx 211 \mathrm{MeV}$, then by interaction this photon with Coulomb field of nuclear can be generate $\mu^{ \pm}$pair. The cross-section for this interaction can obtain from the equation (3.2), replacing electron mass $m_{e}$ on muon mass $m_{\mu}$. This cross-section is less from the cross-section for generation of electron on a value $m_{e} / m \approx(1 / 207)^{2} \approx$ $1.37 \cdot 10^{-5}$, therefore

$$
\sigma_{p \gamma}(h v) \approx 1.29 \cdot 10^{-32} \mathrm{cм}^{2} \quad \text { for } \quad h v \gg 70 \text { Мев. }
$$

\section{Generation of recoil electrons by collision of charged particles.}

The recoil electrons will be generated by the collision of charged particles with other particles. The magnetosphere of collapsing star consists substantially with electrons and protons. The cross-section for the generation of recoil electron with kinetic energy $W_{e}$ by the collision with relativistic protons with energy $E_{p}$ and charge eZ is $[6,7,8]$

$$
\sigma_{p}\left(E_{p}, W_{e}\right) d W_{e} \approx 2 \pi r_{0}^{2} \frac{m_{e} c^{2}}{\beta_{p}^{2} W_{e}^{2}} d W_{e}
$$


Here $\beta_{p}=\left[1-\frac{\left(m_{p} c^{2}\right)^{2}}{E_{1} E_{2}}\right]^{1 / 2}, \quad \beta_{p} c$ - proton velocity in the center-of-mass system.

Numerical value this cross-section is

$$
\sigma_{p}\left(E_{p}, W_{e}\right) d W_{e} \approx 2.55 \cdot 10^{-25} \frac{1}{\beta_{p}^{2} W_{e}^{2}} d W_{e}
$$

Upper limit of energy, which can be pass to recoil-electron by their directly collision with more massive particle with mass $M$ (when $m_{e} \ll M$ ), is $W_{e} \approx p$.. Here $p$ is impulse of the incident particle. As follows from section 2, the initial electrons and protons in the magnetosphere of collapsing star will accelerate to relativistic energy. Connecting with the magnetic fields, the relativistic electron will lose their energy significant fast from proton, and therefore the electron lifetime is significant less from the proton lifetime. As result the initial electrons will fast loss their energy. Instead of the initial electron in magnetosphere will generate the second electrons by the mutual collisions more rapid protons with electrons.

\section{Neutrino generation in magnetosphere of collapsing star.}

As follows from reactions (1) - (11), in magnetosphere of collapsing star the secondary neutrons, protons, positrons and photons will generate as results of the different processes. These particles will generate neutrino by interactions with particles and fields. The main processes of neutrino generation in the neutral nondegenerate plasma are $[6,15,1617,11,18,19,20,21,22,23,24]$ :

(1) electron-atom bremsstrahlung $e^{-}+(Z, A) \rightarrow(Z, A)+v_{e}+\bar{v}_{e}$;

(2) bremsstrahlung in strong magnetic fields;

(3) Photoneutrino radiation $e^{ \pm}+\gamma \rightarrow e^{ \pm}+v_{e}+\bar{v}_{e}$

(4) Plasmons decay $\Gamma \rightarrow v_{e}+\bar{v}_{e}$;

(5) annihilation of pairs $e^{+}+e^{-} \rightarrow v_{e}+\bar{v}_{e}$;

In next subsection we consider these processes in detail.

\subsection{Neutrino emission by electron-atom collision}

Neutrino can be radiated as a result of electron-atom bremsstrahlung, when the free electron collide with nuclear $(Z, A)$, in consequence of the pair neutrinos will radiate $[6,25]$

$$
(x+a)^{n}=\sum_{k=0}^{n}\left(\begin{array}{l}
n \\
k
\end{array}\right) x^{k} a^{n-k}+(Z, A) \rightarrow(Z, A)+v_{e}+\bar{v}_{e}
$$

In consequence of this process neutrino can to pass the energy from unit volume with speed $[19,22,24]$

$$
Q_{v b r}=1.5 \cdot 10^{27} T_{11}^{5.5} \rho_{10}^{2} \text { ерг } \cdot \mathrm{cm}^{-3} \cdot \mathrm{c}^{-1}
$$

Here $T_{11}=T / 10^{11}, \rho_{10}=\rho / 10^{10} \mathrm{~g} / \mathrm{sm}^{3}$.- baryons density in magnetosphere.

Instead of temperature can to use energy from the relation

$$
T=\frac{E}{k}=7.25 \cdot 10^{15} E
$$

Here $E$ measure in $E r g$ 
Substituting (13) into (12), for neutrino luminosity in magnetosphere of collapsing star we obtain

$$
Q_{v b r}=8.09 \cdot 10^{33} E^{5.5} \rho^{2} \mathrm{Erg} \cdot \mathrm{sm}^{-3} \cdot \mathrm{s}^{-1}
$$

On the final phase of gravitational collapse the density in polar regions magnetosphere can to increase in million times in beside the initial density. The electron energy also can to increase in milliard times and to reach the value $E \geq 10^{12} \mathrm{eB}$ [1-5]. For such energy and density the neutrino luminosity in the magnetosphere of collapsing star can to reach the value

$$
Q_{v b r} \geq 8.09 \cdot 10^{53} \mathrm{Erg} \cdot \mathrm{sm}^{-3} \cdot \mathrm{s}^{-1}
$$

Thus, in the magnetosphere of collapsing star will be generated a powerful stream of neutrinos as a result of braking radiation. In the process of collapse density in the magnetosphere and its temperature will significantly increase. On the condition that the magnetosphere mass is not changed by collapse, i.e.

$$
M_{m}=\int \rho d V=\text { const }
$$

for the density baryons in the magnetosphere at different moments can be written

$$
\rho_{0} V_{0}=\rho V
$$

Here $\rho_{0}, V_{0}$ respectively the initial density and volume of magnetosphere.

From here

$$
\rho=\rho_{0} \frac{V_{0}}{V}
$$

For spherically symmetric baryons distribution in the magnetosphere

$$
V=\frac{4}{3} \pi\left(R_{m}-R\right)^{3}
$$

Here $R_{m}, R$ is the radius magnetosphere and the stellar radius. Therefore the density baryons in the magnetosphere

$$
\rho=\rho_{0}\left(\frac{R_{m_{0}}-R_{0}}{R_{m}-R}\right)^{3}
$$

The stellar radius decreases according to the law of free fall during the gravitational collapse. The magnetosphere radius will also decrease, because the magnetic field and magnetosphere tighten to star. This would increase of the density baryons in the magnetosphere and, in accordance with (12), increasing the neutrino luminosity the unit volume of magnetosphere. In addition, during the collapse the particles energy in the magnetosphere also significantly increased as a result of the acceleration in increasing magnetic field. This too will lead to increase in speed generating neutrinos by brake processes.

Substituting (15) into (14), we obtain for the neutrino luminosity in the magnetosphere for the braking radiation

$$
Q_{v b r}=8.09 \cdot 10^{33} E^{5.5} \rho_{0}^{2}\left(\frac{R_{m_{0}}-R_{0}}{R_{m}-R}\right)^{6} \text { ерг } \cdot \mathrm{cm}^{-3} \cdot \mathrm{c}^{-1}
$$


The particles energy during the collapse changes as [1]

$$
E=E_{0}\left(\frac{R_{0}}{R}\right)^{-A_{1}(\theta)}
$$

Substituting this value of energy in (16), we obtain

$$
Q_{v b r} \approx 8.09 \cdot 10^{33} E_{0}^{5.5} \rho_{0}^{2}\left(\frac{R_{m_{0}}-R_{0}}{R_{m}-R}\right)^{6}\left(\frac{R_{0}}{R}\right)^{-5.5 A_{1}(\theta)} \frac{\mathrm{ep}}{\mathrm{cm}^{3} \mathrm{c}}
$$

The power $A_{1}(\theta)$ in the equation (7) varies within $-0.3 \leq A_{1}(\theta) \leq 1.7$ for the relativistic particles and within $-0.6 \leq A_{1}(\theta) \leq 3.4$ for nonrelativistic particles [1].

As we can see, the power is as positive and negative values depending on the angle $\theta$, gaining zero values when $\theta_{1} \approx 0.88, \theta_{2} \approx 2.23, \theta_{3} \approx 4.00, \theta_{4} \approx 5.40$.

We will consider the neutrino luminosity for the star with a mass $M$ and the initial radius $R_{0}$, which collapses to object with radius $R$, which changes during the gravitational collapse according to the free fall law

$$
\frac{d R}{d t}=\left(\frac{2 G M}{R_{0}}\right)^{1 / 2}\left(\frac{R_{0}-R}{R}\right)^{1 / 2}
$$

Here $G$ is the gravitational constant.

Integrating (18), we obtain

$$
\left(\frac{2 G M}{R_{0}}\right)^{1 / 2} t=\frac{1}{2}\left(R_{0}-R\right)^{1 / 2} R^{1 / 2}+R_{0} \operatorname{Arc} \sin \left(\frac{R}{R_{0}}\right)^{1 / 2}
$$

Since $\left(\frac{R}{R_{0}}\right)<1$, then after the series expansion of the functions $\operatorname{Arc} \sin \left(\frac{R}{R_{0}}\right)^{1 / 2}$ we obtain

$$
R=\frac{1}{4}\left(\frac{2 G M}{R_{0}}\right) \frac{t^{2}}{R_{0}}
$$

Substituting (19) (17), we obtain

$$
Q_{v b r} \approx 8.09 \cdot 10^{33} E_{0}^{5.5} \rho_{0}^{2}\left(\frac{R_{m_{0}}-R_{0}}{R_{m}-R}\right)^{6}\left(\frac{2 G M}{4 R_{0}^{2} R_{0}}\right) t^{11 A_{1}(\theta)} \mathrm{Erg} \cdot \mathrm{sm}^{-3} \cdot \mathrm{s}^{-1}
$$

From equation (16), (17) and (20) we can to calculate the neutrino luminosity for the electron-atom bremsstrahlung in the magnetosphere for the various moments of the stellar collapse. In the final stages of collapse the density in the polar areas magnetosphere may increase in the billions times in comparison with the initial density. Energy protons can also increase in the final stages of collapse in billions of times and achieve values $E \geq 10^{14} \mathrm{eV}$. So on the final stages of collapse in the magnetosphere has generated a very strong flow of neutrinos. The most intense formation of neutrinos due to brake the processes will occur at the final stages of collapse, when the temperature and baryons density in the magnetosphere reaches the maximum values. 


\subsection{The neutrino radiation as a result of Urca processes in the magnetosphere}

For the magnetosphere collapsing star, which consists with mostly of electrons, protons and neutrons, the particular interest are the reactions of the electron capture on proton and the reactions of the positrons capture on neutron, so-called urca- process

$$
\begin{aligned}
& e^{-}+p \rightarrow n+v_{e} \\
& e^{+}+n \rightarrow p+\bar{v}_{e}
\end{aligned}
$$

The last two processes are urca - processes that are the main processes in the environment with a small density of nuclei. The neutrino luminosity of the volume unit for urca- processes in the magnetosphere of collapsing star [22]

$$
Q_{v U R C A}=9.0 \cdot 10^{33} T_{11}^{6} \rho_{8} \mathrm{Erg} \cdot \mathrm{sm}^{-3} \cdot \mathrm{s}^{-1} .
$$

Here $T_{11}=T / 10^{11}, \quad \rho_{8}=\rho / 10^{8} \mathrm{r} / \mathrm{CM}^{3}$ - baryons density in the magnetosphere Substituting (13) into (21), we obtain

$$
Q_{v U R C A}=1.31 \cdot 10^{55} E^{6} \rho \mathrm{Erg} \cdot \mathrm{sm}^{-3} \cdot \mathrm{s}^{-1} .
$$

For the magnetosphere of collapsing $\operatorname{star} E=E_{0}\left(\frac{R_{0}}{R}\right)^{-A_{1}(\theta)}$, therefore

$$
Q_{v U R C A}=1.31 \cdot 10^{55} E_{0}^{6}\left(\frac{R_{0}}{R}\right)^{-6 A_{1}(\theta)} \rho \mathrm{Erg} \cdot \mathrm{sm}^{-3} \cdot \mathrm{s}^{-1}
$$

These calculations indicate that the intensity of radiation neutrinos from urca-processes in the magnetosphere is very substantially depends on the energy of the particles. Since the particles energy grow very quickly during collapse, the urca-processes in the magnetosphere will play the significant role in the neutrinos generation during collapse. In the final stages of the collapse the protons in magnetosphere can accelerate to energies $E>10^{16} \mathrm{eV}\left(T>10^{20} \mathrm{~K}\right)$, and the magnetosphere density grow in millions of times. In this case, even the nondensity magnetosphere is a powerful source of neutrinos due to urca- process, because the neutrino luminance very much depends on the protons energy. So, if in the magnetosphere the electron capture on proton or on neutron with energy $E>10^{10}$ $e V\left(T>10^{14} K\right)$, then neutrino will generate with a speed

$$
Q_{v U R C A}>10^{52} \rho_{8} \mathrm{Erg} \cdot \mathrm{sm}^{-3} \cdot \mathrm{s}^{-1}
$$

As a result of high neutrino luminosity due to urca-processes even with the relatively small density protons and neutrons in the magnetosphere the numerous neutrinos can be generated. So, if the nucleons density in the magnetosphere on certain stages of the collapse increase to the value $\rho=$ $10^{8} \mathrm{~g} / \mathrm{sm}^{3}$, then the neutrino luminosity this magnetosphere from urca- processes will exceed the values

$$
Q_{v U R C A}>10^{52} \mathrm{Erg} \cdot \mathrm{sm}^{-3} \cdot \mathrm{s}^{-1} .
$$

This is a very powerful flow of neutrinos, which will be free to come out from the magnetosphere collapsing star. 


\subsection{Neutrino generation due to of electron brake radiation in strong magnetic field}

Neutrinos can be generated also as a result of the synchrotron radiation electron, accelerated in the strong magnetic field. This process is sufficient on the final stages of the stellar collapse when the stellar magnetic field strongly increases. Neutrino luminance for this process for a relativistic degenerate electron gas $[10,11,26]$

$$
Q_{v e B}= \begin{cases}3 \cdot 10^{-44} B_{8}^{6} T_{7} \rho^{4}, & \text { for } B_{8} \rho^{\frac{2}{3}} \leq 8 \cdot 10^{6} T_{7}, \\ 4 \cdot 10^{-7} B_{8}^{\frac{2}{3}} T_{7}^{\frac{19}{3}} \rho^{\frac{4}{9}}, & \text { for } B_{8} \rho^{\frac{2}{3}} \geq 8 \cdot 10^{6} T_{7} .\end{cases}
$$

Here $Q_{v e B}$ is measured in Erg. $s m^{-3} \cdot s^{-1}$.), and the magnitude $B_{8}=B / 10^{8}$.

For the dipole magnetic field in the magnetosphere of collapsing star

$$
B(r, \theta, R)=(1 / 2) F_{0} R r^{-3}\left(1+3 \cos ^{2} \theta\right)^{1 / 2}
$$

Neutrino luminosity by means of the synchrotron radiation

$Q_{v e B}=\left\{\begin{array}{cl}4.7 \cdot 10^{-46} F_{0}^{6} R_{8}^{6} r^{-18} T_{7} \rho^{4}\left(1+3 \cos ^{2} \theta\right)^{3}, & \text { for } B_{8} \rho^{\frac{2}{3}} \leq 8 \cdot 10^{6} T_{7}, \\ 6.2 \cdot 10^{-9} F_{0}^{\frac{2}{3}} R_{8}^{\frac{2}{3}} r^{-2} T_{7}^{\frac{19}{3}} \rho^{\frac{4}{9}}\left(1+3 \cos ^{2} \theta\right)^{\frac{1}{3}}, & \text { for } B_{8} \rho^{\frac{2}{3}} \geq 8 \cdot 10^{6} T_{7} .\end{array}\right.$

Substituting (13) into (25), we obtain

$Q_{v e B}= \begin{cases}3.4 \cdot 10^{-30} F_{0}^{6} R_{8}^{6} r^{-18} E_{7} \rho^{4}\left(1+3 \cos ^{2} \theta\right)^{3}, & \text { for } B_{8} \rho^{\frac{2}{3}} \leq 8 \cdot 10^{6} T_{7}, \\ 1.8 \cdot 10^{7} F_{0}^{\frac{2}{3}} R_{8}^{\frac{2}{3}} r^{-2} E_{7}^{\frac{19}{3}} \rho^{\frac{4}{9}}\left(1+3 \cos ^{2} \theta\right)^{\frac{1}{3}}, & \text { for } B_{8} \rho^{\frac{2}{3}} \geq 8 \cdot 10^{6} T_{7} .\end{cases}$

For the magnetosphere of collapsing star $E=E_{0}\left(\frac{R_{0}}{R}\right)^{-A_{1}(\theta)}$, therefore

$Q_{v e B}=$

$\left\{\begin{array}{l}3.4 \cdot 10^{-30} F_{0}^{6} R_{8}^{6} r^{-18} E_{07}\left(\frac{R_{0}}{R}\right)^{-A_{1}(\theta)} \rho^{4}\left(1+3 \cos ^{2} \theta\right)^{3}, \quad \text { for } B_{8} \rho^{\frac{2}{3}} \leq 8 \cdot 10^{6} T_{7}, \\ 1.8 \cdot 10^{7} F_{0}^{2 / 3} R_{8}^{2 / 3} r^{-2} E_{07}^{19 / 3}\left(\frac{R_{0}}{R}\right)^{-\frac{19}{3} A_{1}(\theta)} \rho^{4 / 9}\left(1+3 \cos ^{2} \theta\right)^{1 / 3}, \text { for } B_{8} \rho^{\frac{2}{3}} \geq 8 \cdot 10^{6} T_{7} .\end{array}\right.$

The neutrino luminosity for star with the initial radius $R_{0}$ and magnetic flux $F_{0}=5.10^{23} \mathrm{Gs} . \mathrm{cm}^{2}$

$Q_{v e B}$
$=\left\{\begin{array}{l}5.3 \cdot 10^{57} E_{0} R^{6} r^{-18}\left(\frac{R_{0}}{R}\right)^{-A_{1}(\theta)} \rho^{4}\left(1+3 \cos ^{2} \theta\right)^{3}, \quad \text { for } B_{8} \rho^{\frac{2}{3}} \leq 8 \cdot 10^{6} T_{7}, \\ 5.9 \cdot 10^{-38} E_{0}^{19 / 3} R^{2 / 3} r^{-2}\left(\frac{R_{0}}{R}\right)^{-\frac{19}{3} A_{1}(\theta)} \rho^{4 / 9}\left(1+3 \cos ^{2} \theta\right)^{1 / 3}, \text { for } B_{8} \rho^{\frac{2}{3}} \geq 8 \cdot 10^{6} T_{7} .\end{array}\right.$ 
As follows from the relationship (28), the neutrino luminosity of the relativistic degenerate electron gas strongly depends on its density and magnitude of the magnetic field. In the final stages of collapse, when the particles density and the magnetic field in the magnetosphere strongly grow, this process can provide a significant contribution to the neutrino luminosity of the magnetosphere.

\subsection{Generation of neutrinos due to photoneutrino processes}

Neutrinos may occur as a result of the collision of photons with electrons in photoneutrino reactions $[27,28,29]$

$$
e^{ \pm}+\gamma \rightarrow e^{ \pm}+v_{e}+\bar{v}_{e}
$$

Neutrino luminosity due to photoneutrino processes in hot plasma with taking into account the contribution electrons and of positrons is determined by the formula [19]

$$
Q_{v \text { photo }}=1.1 \cdot 10^{31} \mathrm{~T}_{11}^{9} \mathrm{Erg} \cdot \mathrm{cm}^{-3} \cdot \mathrm{s}^{-1},
$$

where $T_{11}=T / 10^{11}$.

If the energy $E$ is measured in Ergs, then

$$
Q_{v \text { photo }}=6.09 \cdot 10^{74} E^{9} \mathrm{Erg} \cdot \mathrm{cm}^{-3} \cdot \mathrm{s}^{-1} \text {, }
$$

For charged particles in the magnetosphere collapsing star

$$
E=E_{0}\left(\frac{R_{0}}{R}\right)^{-A_{1}(\theta)}
$$

Therefore the neutrino luminosity in magnetosphere as a result of the photoneutrino processes

$$
Q_{v \text { photo }}=6.09 \cdot 10^{74} E_{0}^{9}\left(\frac{R_{0}}{R}\right)^{-9 A_{1}(\theta)}
$$

As we can see from the value (31), the neutrino luminosity of the charged particles in the magnetosphere strongly depends on its initial energy and the value $\left(\frac{R_{0}}{R}\right)$.

\subsection{Pair neutrino generation by plasmon decay}

When photon move through ionized gas, a virtual electron-hole pair formed, which formed a plasmon. This plasmon may to decay and form a pair neutrino by the reactions

$$
\Gamma \rightarrow v_{e}+\bar{v}_{e}
$$

Neutrino luminosity such a process is determined by the ratio [19]

$$
Q_{v \text { plasma }}=7.1 \cdot 10^{26} T_{11}^{9} \quad \mathrm{erg} \cdot \mathrm{cm}^{-3} \cdot \mathrm{s}^{-1} .
$$

Replacing the temperature on energy through formula (13) we obtain

$$
Q_{v \text { plasma }}=3.93 \cdot 10^{70} E^{9} \mathrm{erg} \cdot \mathrm{cm}^{-3} \cdot \mathrm{s}^{-1} \text {. }
$$


The substitution of the value $E=E_{0}\left(\frac{R_{0}}{R}\right)^{-A_{1}(\theta)}$ into (33) given

$$
Q_{v \text { plasma }}=3.93 \cdot 10^{70} E_{0}^{9}\left(\frac{R_{0}}{R}\right)^{-9 A_{1}(\theta)}
$$

\subsection{Formation of the neutrino couples by annihilation of electron-positron pairs}

In paragraph 3 it has been shown that in the magnetosphere of collapsing star will generated the great number electron-positron pairs. In the annihilation processes of these pairs can be formed the neutrino couples as a result of reactions $[28,30,31]$

$$
e^{+}+e^{-} \rightarrow v_{e}+\bar{v}_{e}
$$

Neutrino luminosity through this process

$$
Q_{v \text { pair }}=3.6 \cdot 10^{33} T_{11}^{9} \quad \mathrm{erg} \cdot \mathrm{cm}^{-3} \cdot \mathrm{s}^{-1},
$$

Replacing the temperature on energy through (13), we obtain

$$
Q_{v \text { pair }}=1.99 \cdot 10^{77} E^{9} \quad \mathrm{erg} \cdot \mathrm{cm}^{-3} \cdot \mathrm{s}^{-1},
$$

Substituting $E=E_{0}\left(\frac{R_{0}}{R}\right)^{-A_{1}(\theta)}$, we obtaining

$$
Q_{v \text { pair }}=1.99 \cdot 10^{77} E_{0}^{9}\left(\frac{R_{0}}{R}\right)^{-9 A_{1}(\theta)} \quad \mathrm{erg} \cdot \mathrm{cm}^{-3} \cdot \mathrm{s}^{-1}
$$

So, there are the many mechanisms for generating neutrino in the magnetosphere collapsing star. Their efficiency depends on the temperature and will vary during the collapse, when the temperature increases the magnetosphere.

\subsection{Generation neutrino by mesons decay}

If the plasma temperature exceeds the amount $610^{11} \mathrm{~K}(E=k T=50 \mathrm{MeV})$, in the field of thermal radiation of stars may occur neutrinos as a result of mesons decay in the magnetosphere collapsing star (see. (1) and (2)):

$$
\begin{gathered}
\pi^{ \pm} \rightarrow \mu^{ \pm}+v_{\mu} / \bar{v}_{\mu}, \\
\mu^{ \pm} \rightarrow e^{ \pm}+v_{e} / \bar{v}_{e}+v_{\mu} / \bar{v}_{\mu} .
\end{gathered}
$$

The energy loss for muon decay [32]

$$
\dot{E}_{\mu}=9.9 \cdot 10^{38} \frac{\exp (-\beta)}{\rho \beta^{\frac{3}{2}}} \operatorname{erg} \cdot g^{-1} \cdot \mathrm{s}^{-1},
$$

Here $\beta=\frac{m_{\mu} c^{2}}{k T}, \quad m_{\mu} c^{2}=105,695 \mathrm{MeV}-$ the rest mass of muon.

Loss of energy due to pìon decay 


$$
\dot{E}_{\pi}=9.6 \cdot 10^{40} \frac{\exp (-\beta)}{\rho \beta^{3 / 2}} \operatorname{erg} \cdot g^{-1} \cdot s^{-1},
$$

Here $\beta=\frac{m_{\pi} c^{2}}{k T}, m_{\pi} c^{2}=139,576 \mathrm{MeV}-$ the rest mass of pion.

The luminosity of the unit wolume by $\mu$-meson decay is defined by the formula [33]

$$
Q_{\nu \mu} \approx 4 \cdot 10^{32} T_{9}^{3}
$$

for $50 \leq T_{9} \leq 500$.

Or

$$
Q_{v \mu} \approx 1.52 \cdot 10^{53} E^{3} \quad \mathrm{erg} \cdot \mathrm{cm}^{-3} \cdot \mathrm{s}^{-1}
$$

As already indicated, the formation of $\pi$-mesons is possible only for very high energy protons (over $290 \mathrm{MeV}$ ). These protons will generate in the magnetosphere collapsing star due to their acceleration in the variable magnetic field, so this mechanism will take place in the magnetosphere star.

\subsection{Neutrino absorption in magnetosphere}

Neutrinos can absorb in the magnetosphere due to their interaction with matter. The interaction crosssection for electron neutrino and muon neutrinos with the substance is very small and is approximately $10^{-44} \mathrm{~cm}^{2}$ for neutrinos with energies $1 \mathrm{MeV}$. The full rate neutrino scattering on electrons, protons or neutrons is given by the formula [20]

$$
\begin{aligned}
& P_{v e} \approx 9.1 \cdot 10^{-35} N_{e} N_{v} T_{9}^{2}\left[c^{-1} \mathrm{cM}^{-3}\right], \\
& P_{v p} \approx 1.5 \cdot 10^{-6} N_{p} N_{v} T_{9}^{5}\left[c^{-1} c \mathrm{M}^{-3}\right] .
\end{aligned}
$$

Here $N_{v}=7.65 \cdot 10^{27} T_{9}^{3}$ - a number of neutrino in the unit of volume, $N_{e}, N_{p}$ - number of protons or neutrons.

\subsection{Numerical evaluation of neutrino luminosity in magnetosphere of collapsing star.}

From the previous sections we can see that in the magnetosphere collapsing star as a result of the various mechanisms the neutrinos flux will generate. Neutrino may freely leave the magnetosphere and go outside. As a result this magnetosphere loses its energy. Rate of energy loss due to neutrino luminosity for different mechanisms can be evaluated by using formulas (14), (22), (24), (28), (30), (31) and (33). As we can see from these formulas, the most efficient generating neutrino processes in the magnetosphere collapsing star is the pair annihilation. Therefore for the neutrino luminosity in magnetosphere collapsing star can written

$$
Q_{v} \approx Q_{v \text { pair }} \approx 1.99 \cdot 10^{77} E^{9} \quad \mathrm{erg} \cdot \mathrm{cm}^{-3} \cdot \mathrm{s}^{-1}
$$

Neutrino luminosity from the all magnetosphere of collapsing star can obtain integrating the specific neutrino luminosity around the all volume of magnetosphere. 


$$
L_{v}=\int Q_{v} d V
$$

here $d V$ - the volume element of magnetosphere.

Because neutrinos not interact practically with magnetic fields, their distribution in the magnetosphere is homogeneous and spherically-symmetric. For such case

$$
d V=4 \pi r^{2} d r
$$

Substituting (43) (42) and integrating by $r$ within $R \leq r \leq R_{m}$, we obtain the full neutrino luminosity for magnetosphere collapsing star

$$
L_{v}=\frac{4}{3} \pi\left(R_{m}-R\right)^{3} Q_{v}
$$

Here $R$ is the radius of star, $R_{m}$ is the radius of magnetosphere.

Substituting (42) (43), we obtain for the full neutrino luminosity in the magnetosphere of collapsing star

$$
L_{v} \approx 8 \cdot 10^{77}\left(R_{m}-R\right)^{3} E^{9}
$$

This formula allows you to evaluate the full neutrino luminosity in the magnetosphere.

As we can see, the magnetosphere of collapsing stars are the very powerfull sources of neutrinos, which are generated by the nuclear reactions and the annihilation of electron-positron pairs.

\section{Conclusion}

The original protons and electrons in magnetosphere are accelerated to relativistic energies during the collapse. Interacting between the particles and magnetic fields, these particles will lose its energy on ionization and radiation. At the same time in the magnetosphere collapsing star will be generated secondary particles, such as electrons, protons, neutron, meson, neutrinos and gamma photons as a result of the different interaction between the particles and fields. These secondary particles too will accelerate in the increasing magnetic field, generating the cascade particles and photons. Cascading interactions between the particles and photons will generate particles and photons. Thus, the magnetosphere of collapsing star will be generated by the multiple cascading interactions particles and their acceleration. It will consist from protons (p), neutrons (n), electrons (e-), positrons $(\mathrm{e}+)$, mezos $(\pi, \mu)(v)$, neutrinos and photons $(\gamma)$.

Some part of the charged particles and the neutrinos will leave the magnetosphere and come in a interstellar medium. This means that the magnetospheres collapsing stars are powerful sources of cosmic rays and neutrinos in our Galaxy and other galaxies. The contribution of these sources in the general flux of cosmic rays and neutrinos will be made in the next work. 


\section{References}

1. V. Kryvdyk, MNRAS, 309, 593 (1999)

2. V. Kryvdyk, Kinem. and Phys. Cel. Bodies, 25, 277 (2010)

3. V. Kryvdyk, MNRAS, 309, 593 (1999)

4. V. Kryvdyk, Adv. Space Res., 28, 463 (2001)

5. V. Kryvdyk, Adv. Space Res., 31, 1315 (2003)

6. K. R. Leng, Astrophysical formulae (Springer-Verlag, Berlin-Heidelberg-New York,1974)

7. S. Hajakawa, Cosmic ray physics. Part 1. Nuclear aspects (1978, Mir, Moskva)

8. S. Hajakawa, Cosmic ray physics. Part 2. Astrophysical aspects (1978, Mir, Moskva)

9. H.Y. Chiu, E.E. Salpeter, Phys. Rev. Let., 12, 413 (1964)

10. V. Canuto, H. Y., Chiu, C.K. Chou, L. Fassio-Canuto, Phys. Rev. D., 2, 281 (1970)

11. L. Fassio -Canuto, Phys. Rev., 187, 2141 (1969)

12. L. Baiotti, B. Giacomazzo, L. Rezzolla, Phys. Rev. D, 78, id. 084033 (2008)

13. B.L. Friman, O.V. Maxwell, Ap. J., 232, 541 (1979)

14. W. Heitel, The quantum theory of radiation (1954, Oxford University Press, Oxford).

15. E. Braaten, D. Segel, Phys. Rev. D, 48, 1478 (1993)

16. S. W. Bruenn, Ap. J. Sup.Ser., 58, 771 (1985)

17. D. A. Dicus, Phys. Rev. D, 6, 941 (1972)

18. N. Itoh, T. Adachi, M. Nakagawa, Y. Kohyama, H. Munakata, Ap. J., 339, 354 (1989)

19. H. B. J. Koers, R. A. M. J. Wijers, MNRAS, 364, 934 (2005)

20. J. M. Lattimer, M. Prakash, C. J. Pethick, P. Haensel, Phys. Rev. Let., 66, 2701 (1991)

21. H. Munakata, Y. Kohyama, N. Itoh, Ap. J., 296, 197, 1985.

22. Y. Z. Qian, S. E. Woosley, Ap. J., 471, 331 (1996)

23. S. Ratkovic, S. I. Dutta, M. Prakash, Phys. Rev. D, 67, id. 123002 (2003)

24. M. M. Reynoso, G. E. Romero, O. A. Sampayo, Astron. Astrophys., 454, 11 (2006)

25. B. M. Pontekorvo, JETP, 36, 1615 (1959)

26. J. D. Landstreet, Phys. Rev., 153, 1372 (1967)

27. V .I. Ritus, JETP, 41, 1285 (1961)

28. H. Y. Chiu, R.C. Stabler, Phys. Rev., 122, 1317 (1961)

29. V. Petrosian, G.Beaudet, E.E. Salpeter, Phys. Rev.,154, 1445 (1967)

30. H. Y. Chiu, Phys. Rev.,123, 1040 (1961)

31. H. Y. Chiu, P. Morrison, Phys. Rev. Let., 5, 573 (1960)

32. W. D. Arnett, Canad. J. Phys. 45, 1621 (1967)

33. C. J. Hansen, Astroph. Space Sci., 1, 499 (1968) 